\title{
Adaptive Feedback Control for Chaos Control and Synchronization for New Chaotic Dynamical System
}

\author{
M. M. El-Dessoky ${ }^{1,2}$ and M. T. Yassen ${ }^{2}$ \\ ${ }^{1}$ Mathematics Department, Faculty of Science, King Abdulaziz University, P.O. Box 80203, \\ Jeddah 21589, Saudi Arabia \\ ${ }^{2}$ Mathematics Department, Faculty of Science, Mansoura University, Mansoura 35516, Egypt
}

Correspondence should be addressed to M. T. Yassen, mtyassen@yahoo.com

Received 8 February 2012; Revised 23 April 2012; Accepted 2 May 2012

Academic Editor: Rafael Martinez-Guerra

Copyright (C) 2012 M. M. El-Dessoky and M. T. Yassen. This is an open access article distributed under the Creative Commons Attribution License, which permits unrestricted use, distribution, and reproduction in any medium, provided the original work is properly cited.

This paper investigates the problem of chaos control and synchronization for new chaotic dynamical system and proposes a simple adaptive feedback control method for chaos control and synchronization under a reasonable assumption. In comparison with previous methods, the present control technique is simple both in the form of the controller and its application. Based on Lyapunov's stability theory, adaptive control law is derived such that the trajectory of the new system with unknown parameters is globally stabilized to the origin. In addition, an adaptive control approach is proposed to make the states of two identical systems with unknown parameters asymptotically synchronized. Numerical simulations are shown to verify the analytical results.

\section{Introduction}

Chaos control and synchronization methods were first addressed by Ott et al. [1] and Pecora and Carroll [2] in the beginning of the decade of 1990. Along with these concepts came the idea of chaotic encryption.

Nowadays, different techniques and methods have been proposed to achieve chaos control and chaos synchronization such as linear and nonlinear feedback control [3-10]. Most of them are based on exactly knowing the system structure and parameters. But in practical situations, some or all of the system's parameters are unknown. Moreover, these parameters change from time to time. Therefore, the derivation of an adaptive controller for the control 


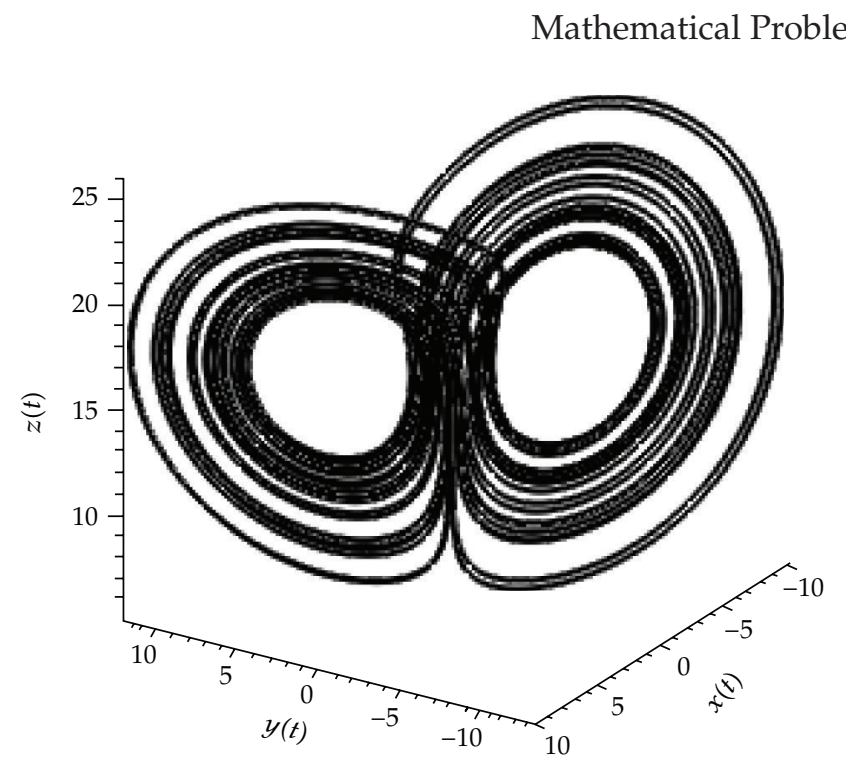

Figure 1: The chaotic attractor of new dynamical system at $a=10, b=16$ and $c=-1$.

and synchronization of chaotic systems in the presence of unknown system parameters is an important issue [11-21].

In recent years, chaos synchronization has received special interests due to its potential applications in secure communications [22-25], biological systems [26], circuits [27], lasers [28], and so forth. In operation, a chaotic system exhibits irregular behavior and produces broadband, noise-like signals, thus, it is thought to use in secure communications.

In this work we investigate the problem of chaos control and synchronization for new chaotic dynamical system and propose a simple adaptive feedback control method for chaos control and synchronization under a reasonable assumption. In comparison with previous methods, the present control technique is simple both in the form of the controller and its application. Based on Lyapunov's stability theory, adaptive control law is derived such that the trajectory of the new system with unknown parameters is globally stabilized to the origin. In addition, an adaptive control approach is proposed to make the states of two identical systems with unknown parameters asymptotically synchronized. Numerical simulations are shown to verify the analytical results.

The object of this work is chaos control and synchronization of two identical new chaotic dynamical systems [29] with adaptive feedback and application in secure communication. The new system [29] is described by

$$
\begin{aligned}
& \dot{x}=a(y-x), \\
& \dot{y}=b x-x z, \\
& \dot{z}=x y+c z,
\end{aligned}
$$

where $a, b$, and $c$ are three unknown parameters. This system exhibits a chaotic attractor at the parameter values $a=10, b=16$, and $c=-1$ (see Figure 1 ).

The paper is organized as follows. In Section 2, we propose the main results of this paper. In Section 3, the adaptive feedback control method is applied to control of new 
attractor with unknown parameters and numerical simulations are presented to show the effectiveness of the proposed method. In Section 4, the adaptive feedback control method is applied to synchronization of two identical new attractor and numerical simulations are presented for verifying the effectiveness of the proposed method. We conclude the paper in Section 5.

\section{Main Results}

In this section, we investigate the problem of chaos control by modifying the previous method [30] and propose the main results of this paper.

Let a chaotic system be given as

$$
\dot{x}=f(x),
$$

where $x=\left(x_{1}, x_{2}, \ldots, x_{n}\right)^{T} \in R^{n}, f(x)=\left(f_{1}(x), f_{2}(x), \ldots, f_{n}(x)\right)^{T}: R^{n} \rightarrow R^{n}$ is a smooth nonlinear vector function. Without loss of the generality, let $x_{e}=0$ is an equilibrium point of the system (2.1). To describe the new design and analysis, the following assumption is needed.

Assumption 2.1. There exists a nonsingular coordinate transformation $y=T x$, such that system (2.1) can be rewritten as

$$
\begin{aligned}
& \dot{z}_{1}=g_{1}\left(z_{1}, z_{2}\right) \\
& \dot{z}_{2}=g_{2}\left(z_{1}, z_{2}\right),
\end{aligned}
$$

where $z_{1}=\left(y_{1}, y_{2}, \ldots, y_{r}\right)^{T} \in R^{r}, z_{2}=\left(y_{r+1}, y_{r+2}, \ldots, y_{n}\right)^{T} \in R^{n-r}$, the second equation satisfies $\dot{z}_{2}=g_{2}\left(0, z_{2}\right)$, with the vector function $g_{2}\left(z_{1}, z_{2}\right)$ being smooth in a neighborhood of $z_{1}=0$, and the subsystem $\dot{z}_{2}=g_{2}\left(0, z_{2}\right)$ is uniformly exponentially stable about the origin $z_{2}=0$ for all $z$.

Remark 2.2. It should be pointed out that not all the finite dimensional chaotic systems are given as (2.2) in their original forms. Therefore, we should make a nonsingular coordinate transformation $T$, which can adjust the array order of the variables $\left(x_{1}, x_{2}, \ldots, x_{n}\right)$ to make the original systems (in the new form) have the form of (2.2). Thus, Assumption 2.1 is reasonable, and system (2.2) is very general, which contains most well-known finite dimensional chaotic systems.

Remark 2.3. The vector function $g_{2}\left(z_{1}, z_{2}\right)$ being smooth in a neighborhood of $z_{1}=0$, that is, there is a positive constant $\lambda_{0}$ (locally) such that $\left\|g_{2}\left(z_{1}, z_{2}\right)-g_{2}\left(0, z_{2}\right)\right\| \leq \lambda_{0}\left\|z_{1}\right\|$. And the subsystem $\dot{z}_{2}=g_{2}\left(0, z_{2}\right)$ is uniformly exponentially stable about the origin $z_{2}=0$ for all $z$, which implies that there are a Lyapunov function $V_{0}\left(z_{2}\right)$ and two positive numbers $\lambda_{1}, \lambda_{2}$ such that

$$
\dot{V}_{0}\left(z_{2}\right)=\frac{\partial V_{0}\left(z_{2}\right)}{\partial z_{2}} g_{2}\left(0, z_{2}\right) \leq-\lambda_{1}\left\|z_{2}\right\|^{2}, \quad\left\|\frac{\partial V_{0}\left(z_{2}\right)}{\partial z_{2}}\right\| \leq \lambda_{2}\left\|z_{2}\right\|,
$$


respectively. Since the system (2.1) is chaotic and $g_{2}\left(z_{1}, z_{2}\right)$ is smooth function, there exists a positive number $\lambda_{3}$, such that $\left\|g_{1}\left(z_{1}, z_{2}\right)\right\| \leq \lambda_{3}\left\|z_{1}\right\|$.

In order to stabilize the chaotic orbits in (2.1) to its equilibrium point $x_{e}=0$, we add the following adaptive feedback controller to system (2.1) and the controlled system (2.1) is as follows:

$$
\begin{gathered}
\dot{z}_{1}=g_{1}\left(z_{1}, z_{2}\right)+u_{1}=g_{1}\left(z_{1}, z_{2}\right)+k_{1} z_{1}, \\
\dot{z}_{2}=g_{2}\left(z_{1}, z_{2}\right)+u_{2}=g_{2}\left(z_{1}, z_{2}\right),
\end{gathered}
$$

where the controller $u=\left(u_{1}, u_{2}\right)^{T}=k_{1} z=\left(k_{1} z_{1}, 0\right)^{T}$. The feedback gain $k_{1}$ is adapted according to the following update law:

$$
\dot{k_{1}}=-\gamma\left\|z_{1}\right\|^{2},
$$

where $\gamma$ is an arbitrary positive constant, in general, we select $\gamma=1$. function

Let the systems (2.2) and (2.4) be the augment systems, and introduce a Lyapunov

$$
V=\frac{1}{2} z_{1}^{T} z_{1}+V_{0}\left(z_{2}\right)+\frac{1}{2 \gamma}\left(k_{1}+L\right)^{2}
$$

where $L=\left(\lambda_{3}+\alpha\right), \alpha \geq \lambda_{0}^{2} \lambda_{1} / 4$. Next, we give the following main result.

Theorem 2.4. Starting from any initial values of the augment system, the orbits of the augment system $\left(x(t), k_{1}(t)\right)^{T}$ converge to $\left(x_{e}, k_{0}\right)^{T}$ as $t \rightarrow \infty$, where $k_{0}$ is a negative constant depending on the initial value. That is to say, the adaptive feedback controller stabilizes the chaotic orbits to its equilibrium point $x_{e}=0$.

Proof. Differentiating the function $V$ along the trajectories of the augment system, we obtain

$$
\begin{aligned}
\dot{V} & =z_{1}^{T} \dot{z}_{1}+\frac{\partial V_{0}\left(z_{2}\right)}{\partial z_{2}} g_{2}\left(z_{1}, z_{2}\right)+\frac{1}{r}\left(k_{1}+L\right) \dot{k_{1}} \\
& =z_{1}^{T}\left(g_{1}\left(z_{1}, z_{2}\right)+k_{1} z_{1}\right)+\frac{\partial V_{0}\left(z_{2}\right)}{\partial z_{2}} g_{2}\left(z_{1}, z_{2}\right)-\left(k_{1}+L\right) z_{1}^{T} z_{1} \\
& =z_{1}^{T} g_{1}\left(z_{1}, z_{2}\right)-L z_{1}^{T} z_{1}+\frac{\partial V_{0}\left(z_{2}\right)}{\partial z_{2}}\left(g_{2}\left(z_{1}, z_{2}\right)-g\left(0, z_{2}\right)\right)+\frac{\partial V_{0}\left(z_{2}\right)}{\partial z_{2}} g\left(0, z_{2}\right) \\
& \leq \lambda_{3} z_{1}^{T} z_{1}-\left(\lambda_{3}+\alpha\right) z_{1}^{T} z_{1}+\lambda_{0} \lambda_{2}\left\|z_{1}\right\|\left\|z_{2}\right\|-\lambda_{1}\left\|z_{1}\right\|^{2} \\
& \leq-\alpha z_{1}^{T} z_{1}+\lambda_{0} \lambda_{2}\left\|z_{1} z_{2}\right\|-\lambda_{1}\left\|z_{2}\right\|^{2} \leq 0 .
\end{aligned}
$$

Obviously, $\dot{V}=0$ if and only if $z_{i}=0, i=1,2$, then the set $E=\left\{\left(z, k_{1}\right) \mid \dot{V}(z)=0\right\}=\{0\}$ is the largest invariant set for the augment system. According to the well-known LaSalle invariance principle, $z_{i}=0, i=1,2$, which implies that $x_{i}=0, i=1,2, \ldots, n$, thus, Theorem 2.4 is obtained. 
Remark 2.5. In general, $n-r \geq 1$, where $n-r$ is the dimension of the variable $z_{2}$. One the other hand, we stabilize the first subsystem $\dot{z}_{1}=g_{1}\left(z_{1}, z_{2}\right)$ by applying the previous method [30]. Therefore, the controllers obtained in this paper are simpler than those controllers obtained by the previous method in general case or the same to those controllers obtained by the previous method even in the worst case $n-r=0$. Accordingly, the present method is a modification of the previous method.

Remark 2.6. If $x_{e} \neq 0$ is an equilibrium point of the chaotic system (1.1), then we make the coordinate transformation $y=x-x_{e}$, which make the original chaotic system (1.1) with new variable $y=\left(y_{1}, y_{2}, \ldots, y_{n}\right)$ has the equilibrium point $y_{e}=0$. That is to say, this method can be also easily utilized whatever $x_{e}$ is origin or not.

\section{Adaptive Feedback Control Method for Controling New Attractor}

In this section, we apply the above technique to control the new chaotic system [29]. Now, we rewrite system (1.1) as the following:

$$
\begin{aligned}
& \dot{x}_{1}=a\left(x_{2}-x_{1}\right), \\
& \dot{x}_{2}=b x_{1}-x_{1} x_{3}, \\
& \dot{x}_{3}=x_{1} x_{2}+c x_{3} .
\end{aligned}
$$

It is easy to know the fact that if $x_{2}=0$ the following two dimensional subsystem of the system (3.1):

$$
\begin{aligned}
& \dot{x}_{1}=-a x_{1} \\
& \dot{x}_{3}=c x_{3},
\end{aligned}
$$

which is uniformly exponentially stable about the origin $x_{1}=0, x_{3}=0$ for all $x_{1}, x_{3}$, then there exists a nonsingular coordinate transformation $y=T x$, that is, $y_{1}=x_{2}, y_{2}=x_{1}, y_{3}=x_{3}$, which can make system (3.1) with new variable $y$ has the form of system (2.2), and the new system with controller $u=k_{1} y=\left(k_{1} y_{1}, 0,0\right)^{T}$ is

$$
\begin{aligned}
& \dot{y}_{1}=b y_{2}-y_{2} y_{3}+k_{1} y_{1}, \\
& \dot{y}_{2}=a\left(y_{1}-y_{2}\right), \\
& \dot{y}_{3}=y_{1} y_{2}+c y_{3}, \\
& \dot{k_{1}}=-r\left\|y_{1}\right\|^{2} .
\end{aligned}
$$


Now, we define a Lyapunov function as

$$
V=\frac{1}{2}\left(y_{1}^{2}+y_{2}^{2}+y_{3}^{2}\right)+\frac{1}{2 \gamma}\left(k_{1}+L\right)^{2},
$$

where $L$ is a sufficiently big constant. It is clear that the Lyapunov function $V(e)$ is a positive definite function. Now, taking the time derivative of (3.4), then we get

$$
\begin{aligned}
\frac{d V(e)}{d t} & =y_{1} \dot{y}_{1}+y_{2} \dot{y}_{2}+y_{3} \dot{y}_{3}+\frac{1}{r}\left(k_{1}+L\right) \dot{K}_{1} \\
& =y_{1}\left(b y_{2}-y_{2} y_{3}+k_{1} y_{1}\right)+y_{2}\left(a y_{1}-a y_{2}\right)+y_{3}\left(y_{1} y_{2}+c y_{3}\right)-\left(k_{1}+L\right) y_{1}^{2} \\
& =b y_{2} y_{1}-y_{1} y_{2} y_{3}+k_{1} y_{1}^{2}+a y_{1} y_{2}-a y_{2}^{2}+y_{3} y_{1} y_{2}+c y_{3}^{2}-k_{1} y_{1}^{2}-L y_{1}^{2} \\
& =(a+b) y_{2} y_{1}-a y_{2}^{2}+c y_{3}^{2}-L y_{1}^{2} \\
& =-\left(a y_{2}^{2}-(a+b) y_{2} y_{1}+L y_{1}^{2}\right)+c y_{3}^{2} \\
& \leq-\left(a y_{2}^{2}-(a+b)\left\|y_{2}\right\|\left\|y_{1}\right\|+L y_{1}^{2}\right)+c y_{3}^{2} \leq-e^{T} P e<0
\end{aligned}
$$

where $e=\left[\left|y_{1}\right|,\left|y_{2}\right|,\left|y_{3}\right|\right]^{T}$ is the states vector, and

$$
P=\left[\begin{array}{ccc}
L & \frac{a+b}{2} & 0 \\
\frac{a+b}{2} & a & 0 \\
0 & 0 & c
\end{array}\right]
$$

Obviously, to ensure that the origin of the system (3.1) is asymptotically stable, the matrix $P$ should be positive definite, which implies that $\dot{V}$ is negative definite under the condition $L \geq(a+b)^{2} / 4 a$ then $d V(e) / d t \leq 0$. According to Theorem 2.4, the origin of system (3.3) is asymptotically stable.

\subsection{Numerical Results}

By using Maple 13 to solve the systems of differential equation (3.1) with the parameters are chosen to $a=10, b=16$, and $c=-1$ in all simulations so that the new system exhibits a chaotic behavior if no control is applied (see Figure 1). The initial states of system (3.1) are $y_{1}(0)=1.5, y_{2}(0)=-2$, and $y_{3}(0)=3.2$ and the initial value of the controller $k_{1}(0)=-1$. 


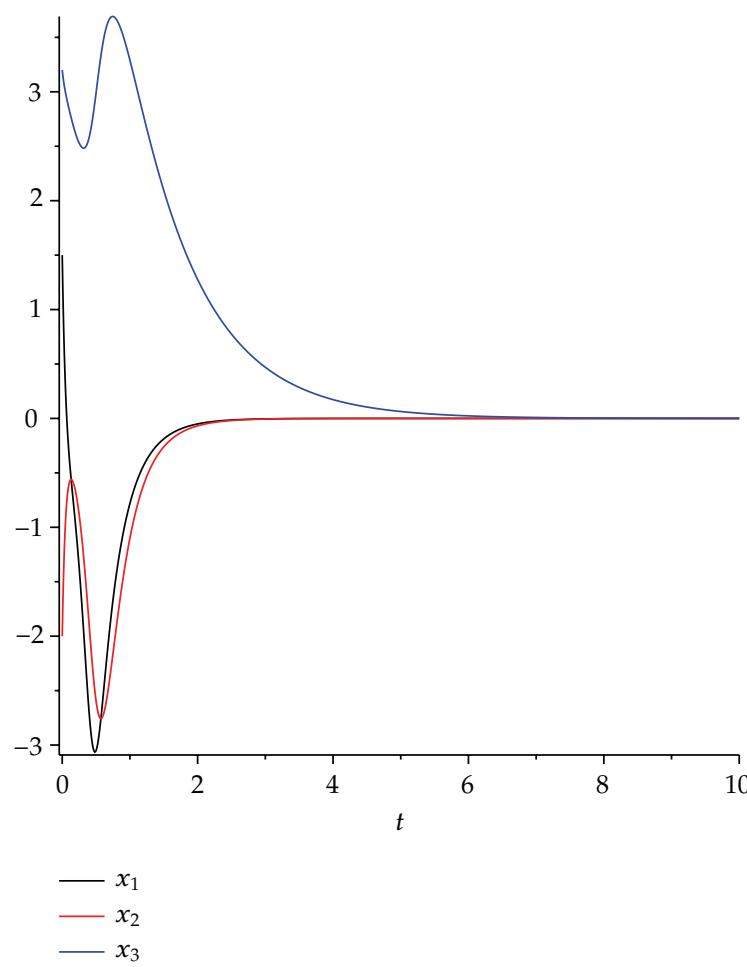

Figure 2: The new dynamical system (3.1) is driven to its stable equilibrium $(0,0,0)$ asymptotically as $t \rightarrow \infty$.

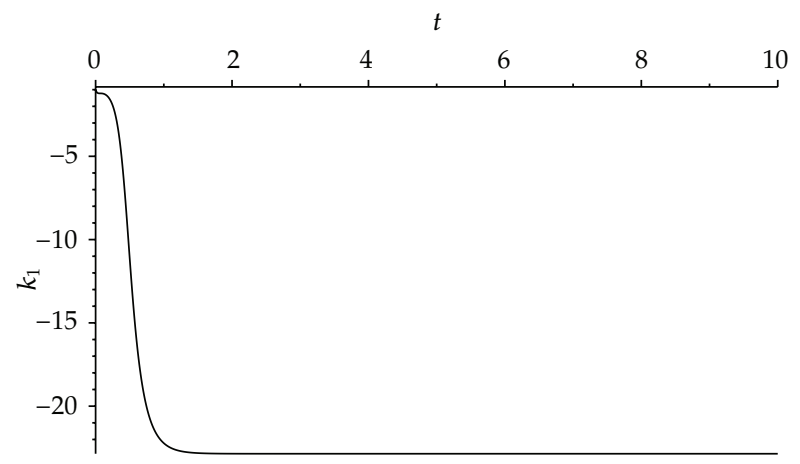

Figure 3: The feedback gain $k_{1}$ tends to a negative constant as $t \rightarrow \infty$.

When $\gamma=1$, the new system is driven to its stable equilibrium $(0,0,0)$ asymptotically as $t \rightarrow \infty$ are shown in Figure 2. The feedback gain $k_{1}$ tends to a negative constant as shown in Figure 3. 


\section{Adaptive Feedback Control Method for Synchronization of Two Identical New Attractors}

In this section, we apply the adaptive feedback control technique for synchronization of two identical new chaotic systems [29]. For the new system (1.1), the master (or drive) and slave (or response) systems are defined below, respectively,

$$
\begin{aligned}
& \dot{x}_{1}=a\left(y_{1}-x_{1}\right), \\
& \dot{y}_{1}=b x_{1}-x_{1} z_{1}, \\
& \dot{z}_{1}=x_{1} y_{1}+c z_{1}, \\
& \dot{x}_{2}=a\left(y_{2}-x_{2}\right) \\
& \dot{y}_{2}=b x_{2}-x_{2} z_{2} \\
& \dot{z}_{2}=x_{2} y_{2}+c z_{2} .
\end{aligned}
$$

For this purpose, the error dynamical system between the drive system (4.1) and response system (4.2) can be expressed by

$$
\begin{gathered}
\dot{x}_{3}=a\left(y_{3}-x_{3}\right) \\
\dot{y}_{3}=b x_{3}-x_{2} z_{3}-z_{1} x_{3} \\
\dot{z}_{3}=c z_{3}+x_{2} y_{3}+y_{1} x_{3}
\end{gathered}
$$

where $x_{3}=x_{2}-x_{1}, y_{3}=y_{2}-y_{1}, z_{3}=z_{2}-z_{1}$.

In order that two chaotic systems can be synchronized in the sense of PS, the following condition should be satisfied:

$$
\lim _{t \rightarrow \infty}\left\|x_{2}-x_{1}\right\|=\lim _{t \rightarrow \infty}\left\|y_{2}-y_{1}\right\|=\lim _{t \rightarrow \infty}\left\|z_{2}-z_{1}\right\|=0
$$

It is easy to know the fact that if $y_{3}=0$ the following two-dimensional subsystem of system (4.3):

$$
\begin{gathered}
\dot{x}_{3}=-a x_{3}, \\
\dot{z}_{3}=c z_{3}+y_{1} x_{3},
\end{gathered}
$$

which is uniformly exponentially stable about the origin $x_{3}=0, z_{3}=0$ for all $x_{3}, z_{3}$, then there exists a nonsingular coordinate transformation $e_{x}=y_{3}, e_{y}=x_{3}, e_{z}=z_{3}$, which can 
make system (4.3) with new variable $e$ has the form of system (2.2), and the new system with controller $u=k_{2} e=\left(k_{2} e_{x}, 0,0\right)^{T}$ is

$$
\begin{gathered}
\dot{e}_{x}=b e_{y}-x_{2} e_{z}-z_{1} e_{y}+k_{2} e_{x}, \\
\dot{e}_{y}=a\left(e_{x}-e_{y}\right), \\
\dot{e}_{z}=c e_{z}+x_{2} e_{x}+y_{1} e_{y}, \\
\dot{k_{2}}=-\gamma\left\|e_{x}\right\|^{2} .
\end{gathered}
$$

Let us consider the Lyapunov function $V(e)$ which is defined by

$$
V(e)=\frac{1}{2}\left(e_{x}^{2}+e_{y}^{2}+e_{z}^{2}+\frac{1}{\gamma}\left(k_{2}+L\right)^{2}\right)
$$

where $L$ is a sufficiently big constant. It is clear that the Lyapunov function $V(e)$ is a positive definite function. Now, taking the time derivative of (4.7), then we get

$$
\begin{aligned}
\frac{d V(e)}{d t}= & e_{x} \dot{e}_{x}+e_{y} \dot{e}_{y}+e_{z} \dot{e}_{z}+\frac{1}{r}\left(k_{2}+L\right) \dot{k}_{2} \\
= & e_{x}\left(b e_{y}-x_{2} e_{z}-z_{1} e_{y}+k_{2} e_{x}\right)+e_{y}\left(a e_{x}-a e_{y}\right) \\
& +e_{z}\left(c e_{z}+x_{2} e_{x}+y_{1} e_{y}\right)-\left(k_{2}+L\right) e_{x}^{2} \\
= & b e_{x} e_{y}-x_{2} e_{x} e_{z}-z_{1} e_{x} e_{y}+k_{2} e_{x}^{2}+a e_{x} e_{y}-a e_{y}^{2} \\
& +c e_{z}^{2}+x_{2} e_{x} e_{z}+y_{1} e_{y} e_{z}-k_{2} e_{x}^{2}-L e_{x}^{2} \\
= & -L e_{x}^{2}-a e_{y}^{2}+c e_{z}^{2}+\left(a+b-z_{1}\right) e_{x} e_{y}+y_{1} e_{y} e_{z} \\
= & -\left[L e_{x}^{2}+\left(z_{1}-a-b\right) e_{x} e_{y}+a e_{y}^{2}-c e_{z}^{2}-y_{1} e_{y} e_{z}\right] \\
\leq & -\left[L e_{x}^{2}+\left(z_{1}-a-b\right)\left\|e_{x}\right\|\left\|e_{y}\right\|+a e_{y}^{2}-c e_{z}^{2}-y_{1}\left\|e_{y}\right\|\left\|e_{z}\right\|\right]=-e^{t} A e \leq 0,
\end{aligned}
$$

where $e=\left[\left|e_{x}\right|,\left|e_{y}\right|,\left|e_{z}\right|\right]^{T}$ is the states vector, and

$$
A=\left[\begin{array}{ccc}
-L & \frac{a+b-z_{1}}{2} & 0 \\
\frac{a+b-z_{1}}{2} & -a & -\frac{y_{1}}{2} \\
0 & -\frac{y_{1}}{2} & c
\end{array}\right]
$$

under the condition $L>\left(z_{1}-a-b\right)^{2} / 4 a$, then $d V(e) / d t \leq 0$. Based on Lyapunov's stability theory, this translates to $\lim _{t \rightarrow \infty}\|e(t)\|=0$. Thus, the response system and drive systems are asymptotically synchronized by using adaptive feedback control method. According to Theorem 2.4, the origin of system (4.6) is asymptotically stable. 


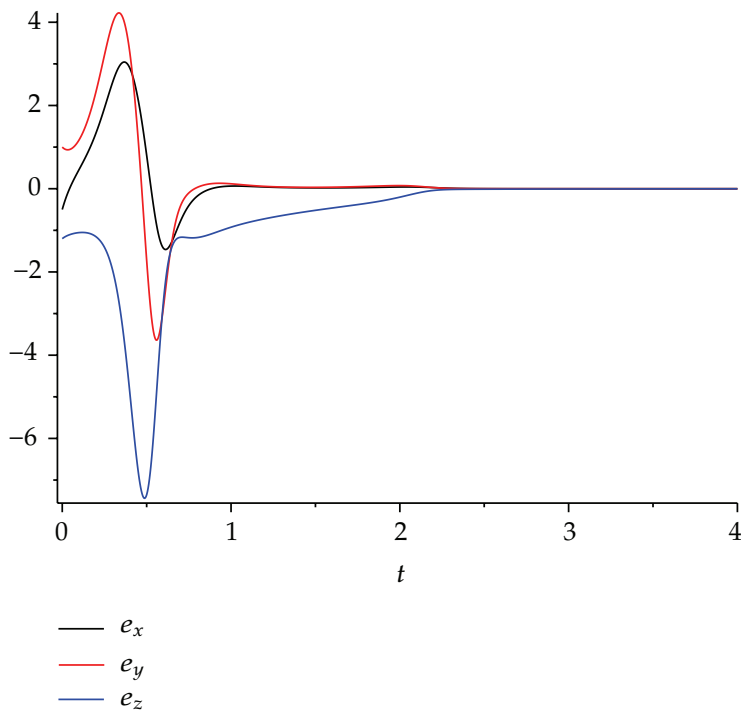

Figure 4: The dynamics of synchronization errors states $e_{x}, e_{y}$, and $e_{z}$ of two identical new dynamical systems with adaptive feedback control.

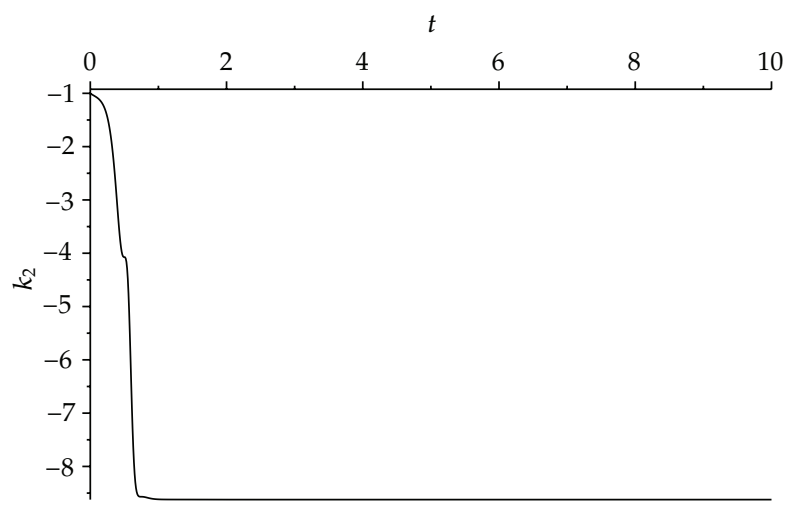

Figure 5: The feedback gain $k_{2}$ tends to a negative constant as $t \rightarrow \infty$.

\subsection{Numerical Results}

By using Maple 13 to solve the systems of differential (4.1), (4.2), and (4.6) with the parameters are chosen to $a=10, b=16$ and $c=-1$ in all simulations, so that the new system exhibits a chaotic behavior if no control is applied (see Figure 1). The initial states of the drive system are $x_{1}(0)=1.5, y_{1}(0)=-2$, and $z_{1}(0)=3.2$, the initial values of the response system are $x_{2}(0)=1, y_{2}(0)=-1$, and $z_{2}(0)=2$, and the initial value of the controller $k_{2}(0)=-1$. When $\gamma=1$, the new system is driven to asymptotically synchronize as $t \rightarrow \infty$ are shown in Figure 4 . The feedback gain $k_{2}$ tends to a negative constant as shown in Figure 5. 


\section{Conclusions}

In this paper, we present a simple adaptive feedback control method for chaos control and synchronization by modifying the previous method. Adaptive feedback control method is applied to control and synchronization of new chaotic dynamical system with known parameters. Numerical simulations are also given to validate the proposed synchronization approach.

\section{Acknowledgment}

The authors would like to thank the Editor and the anonymous reviewers for their constructive comments and suggestions to improve the quality of the paper.

\section{References}

[1] E. Ott, C. Grebogi, and J. A. Yorke, "Controlling chaos," Physical Review Letters, vol. 64, no. 11, pp. 1196-1199, 1990.

[2] L. M. Pecora and T. L. Carroll, "Synchronization in chaotic systems," Physical Review Letters, vol. 64, no. 8, pp. 821-824, 1990.

[3] T. L. Carroll and L. M. Pecora, "Synchronizing chaotic circuits," IEEE Transactions on Circuits and Systems, vol. 38, no. 4, pp. 453-456, 1991.

[4] Y. Zeng and S. N. Singh, "Adaptive control of chaos in Lorenz systems," Dynamics and Control, vol. 7, no. 2, pp. 143-154, 1997.

[5] M. Y. Chen, Z. Z. Han, Y. Shang, and G. D. Zong, "Controlling uncertain van der Pol oscillator via robust nonlinear feedback control," International Journal of Bifurcation and Chaos in Applied Sciences and Engineering, vol. 14, no. 5, pp. 1671-1681, 2004.

[6] J. H. Park, "Controlling chaotic systems via nonlinear feedback control," Chaos, Solitons and Fractals, vol. 23, no. 3, pp. 1049-1054, 2005.

[7] M. T. Yassen, "Chaos control of chaotic dynamical systems using backstepping design," Chaos, Solitons and Fractals, vol. 27, no. 2, pp. 537-548, 2006.

[8] C. Tao and X. Liu, "Feedback and adaptive control and synchronization of a set of chaotic and hyperchaotic systems," Chaos, Solitons and Fractals, vol. 32, no. 4, pp. 1572-1581, 2007.

[9] C. Zhu, "Controlling hyperchaos in hyperchaotic Lorenz system using feedback controllers," Applied Mathematics and Computation, vol. 216, no. 10, pp. 3126-3132, 2010.

[10] G. Chen, "Controlling chaotic and hyperchaotic systems via a simple adaptive feedback controller," Computers and Mathematics with Applications, vol. 61, no. 8, pp. 2031-2034, 2011.

[11] M. Di Bernardo, "An adaptive approach to the control and synchronization of continuous-time chaotic systems," International Journal of Bifurcation and Chaos in Applied Sciences and Engineering, vol. 6, no. 3, pp. 557-568, 1996.

[12] C. W. Wu, T. Yang, and L. O. Chua, "On adaptive synchronization and control of nonlinear dynamical systems," International Journal of Bifurcation and Chaos in Applied Sciences and Engineering, vol. 6, no. 3, pp. 455-471, 1996.

[13] T. L. Liao and S. H. Lin, "Adaptive control and synchronization of Lorenz systems," Journal of the Franklin Institute, vol. 336, no. 6, pp. 925-937, 1999.

[14] E. W. Bai and K. E. Lonngren, "Sequential synchronization of two Lorenz systems using active control," Chaos, Solitons and Fractals, vol. 11, no. 7, pp. 1041-1044, 2000.

[15] H. N. Agiza and M. T. Yassen, "Synchronization of Rossler and Chen chaotic dynamical systems using active control," Physics Letters A, vol. 278, no. 4, pp. 191-197, 2001.

[16] M. C. Ho and Y. C. Hung, "Synchronization of two different systems by using generalized active control," Physics Letters A, vol. 301, no. 5-6, pp. 424-428, 2002.

[17] L. Huang, R. Feng, and M. Wang, "Synchronization of chaotic systems via nonlinear control," Physics Letters A, vol. 320, no. 4, pp. 271-275, 2004. 
[18] J. H. He, Y. Wu, and N. Pang, "Synchronization between two chaotic systems with different order by using active control," International Journal of Nonlinear Sciences and Numerical Simulation, vol. 6, no. 3, pp. 243-248, 20058.

[19] M. T. Yassen, “Controlling chaos and synchronization for new chaotic system using linear feedback control," Chaos, Solitons and Fractals, vol. 26, no. 3, pp. 913-920, 2005.

[20] F. B. Bao and J.-Z. Lin, "Adaptive synchronization of a four-dimensional chaotic system with uncertain parameters," International Journal of Nonlinear Sciences and Numerical Simulation, vol. 6, no. 3, pp. 295-304, 2005.

[21] E. M. Elabbasy and M. M. El-Dessoky, "Adaptive feedback control for the projective synchronization of the Lü dynamical system and its application to secure communication," Chinese Journal of Physics, vol. 48, no. 6, pp. 863-872, 2010.

[22] T. L. Liao and S. H. Tsai, "Adaptive synchronization of chaotic systems and its application to secure communications," Chaos, Solitons and Fractals, vol. 11, no. 9, pp. 1387-1396, 2000.

[23] C. Hua, B. Yang, G. Ouyang, and X. Guan, "A new chaotic secure communication scheme," Physics Letters A, vol. 342, no. 4, pp. 305-308, 2005.

[24] X. Y. Wang and M. J. Wang, "A chaotic secure communication scheme based on observer," Communications in Nonlinear Science and Numerical Simulation, vol. 14, no. 4, pp. 1502-1508, 2009.

[25] J. Lu, X. Wu, and J. Lu, "Synchronization of a unified chaotic system and the application in secure communication," Physics Letters A, vol. 305, no. 6, pp. 365-370, 2002.

[26] S. K. Han, C. Kurrer, and Y. Kuramoto, "Dephasing and bursting in coupled neural oscillators," Physical Review Letters, vol. 75, no. 17, pp. 3190-3193, 1995.

[27] K. M. Cuomo and A. V. Oppenheim, "Circuit implementation of synchronized chaos with applications to communications," Physical Review Letters, vol. 71, no. 1, pp. 65-68, 1993.

[28] R. Roy and K. S. Thornburg, "Experimental synchronization of chaotic lasers," Physical Review Letters, vol. 72, no. 13, pp. 2009-2012, 1994.

[29] W. Zhou, Y. Xu, H. Lu, and L. Pan, “On dynamics analysis of a new chaotic attractor," Physics Letters A, vol. 372, no. 36, pp. 5773-5777, 2008.

[30] R. W. Guo and U. E. Vincent, "Control of a unified chaotic system via single variable feedback," Chinese Physics Letters, vol. 26, no. 9, Article ID 090506, 2009. 


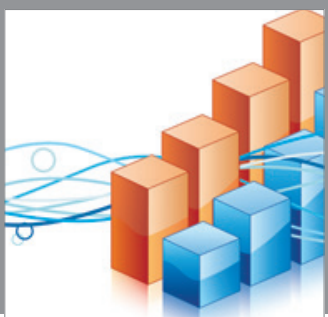

Advances in

Operations Research

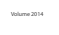

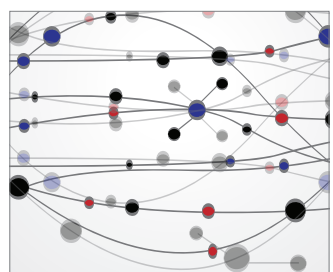

\section{The Scientific} World Journal
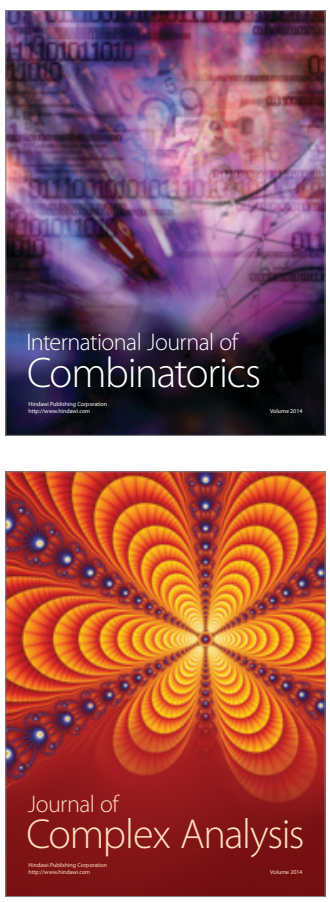

International Journal of

Mathematics and

Mathematical

Sciences
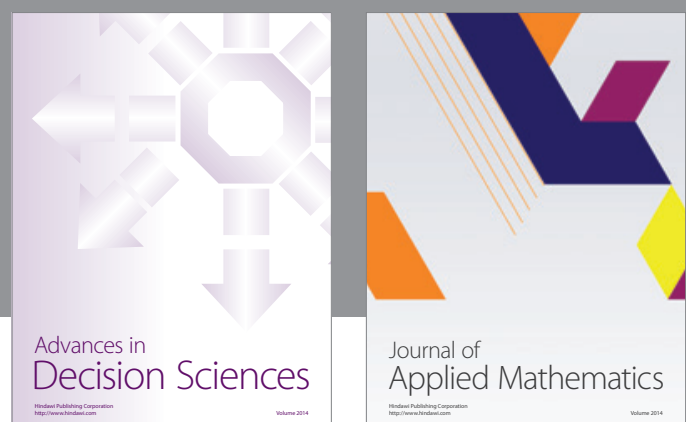

Journal of

Applied Mathematics
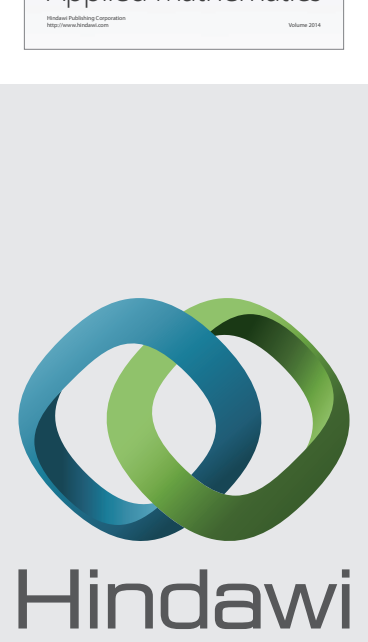

Submit your manuscripts at http://www.hindawi.com
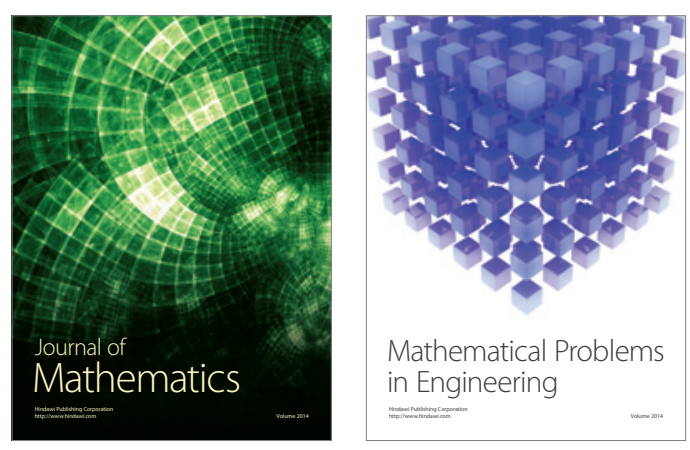

Mathematical Problems in Engineering
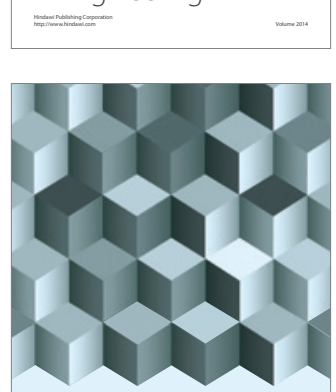

Journal of

Function Spaces
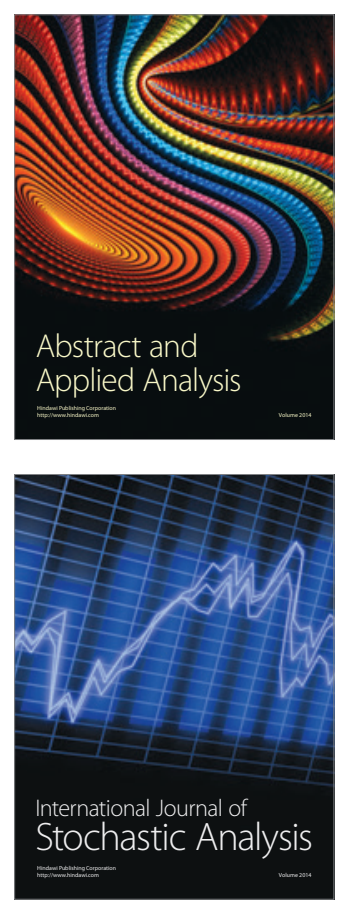

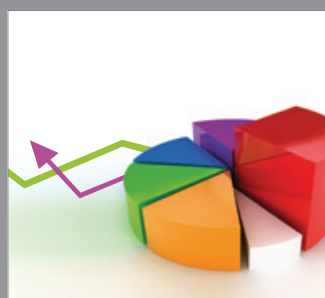

ournal of

Probability and Statistics

Promensencen
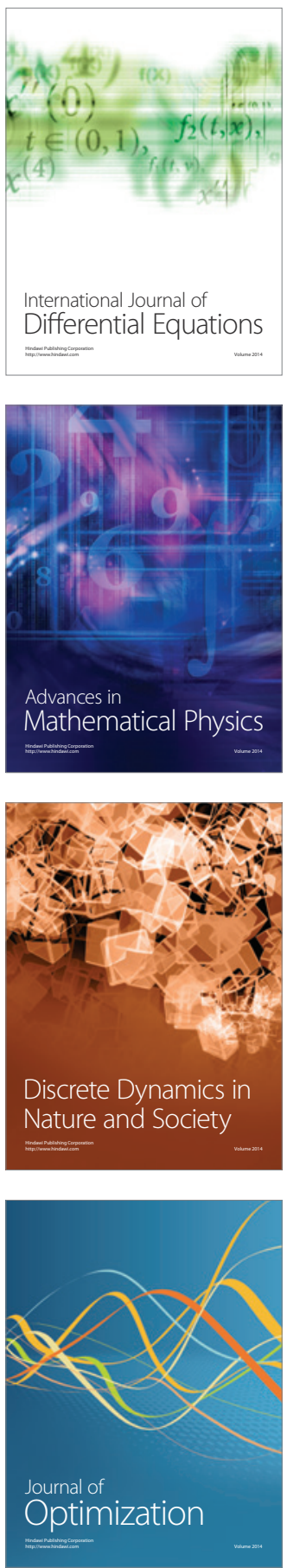